International Journal of Pure and Applied Mathematics

Volume 112 No. $3 \quad 2017,599-604$

ISSN: 1311-8080 (printed version); ISSN: 1314-3395 (on-line version)

url: http://www.ijpam.eu

doi: 10.12732/ijpam.v112i3.11

\title{
NUMERICAL SOLUTION OF OPTIMAL CONTROL PROBLEMS BY THE METHOD OF SUCCESSIVE APPROXIMATIONS
}

\author{
Igor Grigoryev $^{1}$ §, Svetlana Mustafina ${ }^{2}$, Oleg Larin $^{3}$ \\ 1,2 Bashkir State University \\ 32, Validy Str., 450076, Ufa, RUSSIA \\ ${ }^{3}$ South-West State University \\ 94, October st., 305040, Kursk, RUSSIA
}

\begin{abstract}
The paper contains successive approximation method for the solution of optimal control problems. This method is based on Pontryagin's maximum principle. Numerical example of applications of the method for the calculation of optimal trajectories is given.
\end{abstract}

AMS Subject Classification: 49M30, 65K05, 65K10

Key Words: optimal control, optimization methods, method of successive approximations, min-h methods, numerical solution

\section{Introduction}

It's characteristic of the latest technology and highly mechanized and automated production to select the best program of action, the most efficient use of available resources. The objects dealt with technology and production are usually equipped with a "control system" aimed at controlling actions. Mathematically, the behavior of the object is described by certain equations including the control parameters defining a "control system" position [3]. Naturally, there is a question of finding out the best (optimal) to some extent action control. This issue is a problem of an optimal control.

Received: $\quad$ November 21, 2016

Revised: $\quad$ December 29, 2016

Published: $\quad$ February 9, 2017

(C) 2017 Academic Publications, Ltd. url: www.acadpubl.eu

$\S_{\text {Correspondence author }}$ 
The main result of the optimal control theory is Pontryagin's maximum principle setting a general prerequisite for an optimal control. It allows to search for some control actions not referring to the continuous functions class, or when some inequality constraints including problems with phase constraints are imposed on variables problems.

Due to the constantly developing computing capacities, it is natural to work out not so analytical as numerical methods to solve optimal control problems. In this paper we consider the optimization in performance which is one of the most important and common tasks in the optimal control theory, and also both analytical and numerical algorithms as methods for solving such problems.

\section{Problem Statement}

Consider the following optimal control problem:

$$
\text { minimize } I(u)=\int_{t_{0}}^{T} f^{0}(t, x(t), u(t)) d t
$$

subject to

$$
\begin{gathered}
\frac{d x_{i}}{d t}=f_{i}(t, x(t), u(t)), t \in\left[t_{0}, T\right], x(0)=x_{0}, \\
\phi(u) \leq 0
\end{gathered}
$$

where $u(t) \in R$ is the function characterizing the operating influence, $x(t) \in R^{n}$ is function describing a condition of process and $t$ is time.

\section{Method of Successive Approximations}

The optimum control task (8) - (3) can be reduced to the solution of a boundary value problem of the differential equations system of the 2 nd order by means of the maximum principle.

Let's introduce $n$ - dimensional vector $\psi=\left(\psi_{1}, \psi_{2}, \ldots, \psi_{n}\right)$ of conjugate variables and Hamilton's function $H$ :

$$
H(t, x, \psi, u)=\psi^{T} f(t, x, u) .
$$

Let's write down the conjugate system:

$$
\frac{d \psi_{i}}{d t}=-\sum_{j=1}^{n} \frac{\partial f_{j}(t, x(t), u(t))}{\partial x_{i}}, i=1 \ldots n .
$$


with boundary conditions:

$$
\psi(T)=-\frac{\partial F(x(T))}{\partial x} .
$$

According to the maximum principle the required optimum control delivers functions $H(t, x, \psi, u)$ at most on $u \in U$ at any $t \in\left[t_{0}, T\right]$, if $x(t)$ and $\psi(t)$ satisfy system 8 and boundary conditions 6 .

One of the most widespread methods of solution of the boundary value problem is the method of successive approximations in the controls area [2]. The method of successive approximations for numerical solution of optimal control problems is based on Pontryagin's maximum principle. This method consists of successive integrations of the state and costate systems and in changes of the control functions which are defined by maximizing the Hamiltonian.

We specify an initial approximation for control $u^{0}(t)$ (its choice can be based on any physical reasons) and set a counter of iterations number equal to 0 .

The method is iterative and $k$ iteration consist in the following:

1. Let's integrate the control system with the control $u=u^{k}(t)$ to the moment $t=T$. In this connection, the trajectory $x^{k}(t)$ and boundary conditions for the conjugate system are determined.

2. Let's integrate the conjugate system from the moment $t=T$ to $t=t_{0}$ on $u=u^{k}(t), x=x^{k}(t)$ - let's determine the conjugate variables $\psi^{k}(t)$ on the interval $\left[t_{0}, T\right]$.

3. Let's determine the new approximation $u=u^{k+1}(t)$ on the interval $\left[t_{0}, T\right]$ from the function maximum

$$
\begin{aligned}
& H\left(t, x^{k}(t), \psi^{k}(t), u^{k+1}(t)\right)= \\
& \max _{u \in U} H\left(t, x^{k}(t), \psi^{k}(t), u^{k}(t)\right) .
\end{aligned}
$$

4. If condition 7 determines $u=u^{k+1}(t)$ in a not unique way, we choose any of possible values. Then we pass to the next iteration and etc.

If the process of successive approximations converges, we continue it until the further approximations differ from each other within the required accuracy. The decision obtained after the convergence will satisfy the maximum principle. It is necessary to note that convergence of the iterative process significantly depends on the choice of the first approximation. 


\section{Discussion}

The software for the numerical calculations presented below in this article was developed in Borland Delphi environment. In the following example we compute the absolute errors in the following specified norms [1]:

$$
\varepsilon_{x}=\sqrt{\sum_{i}\left(x_{i}-x^{*}\left(t_{i}\right)\right)^{2}}, \varepsilon_{u}=\sqrt{\sum_{i}\left(u_{i}-u^{*}\left(t_{i}\right)\right)^{2}} .
$$

Example. Consider the following optimal control system:

$$
\begin{gathered}
\left\{\begin{array}{l}
\dot{x_{1}}(t)=x_{2}(t), \\
\dot{x_{2}}(t)=-x_{1}(t)+u(t) ;
\end{array}\right. \\
x_{1}(0)=0, x_{2}(0)=0 ;|u| \leq 1 ; t \in[0,2 \pi] .
\end{gathered}
$$

The performance measure is:

$$
I\left(x_{1}, x_{2}\right)=x_{2}(2 \pi) \rightarrow \min .
$$

The optimal control problem is to find a control law $u^{*}(\cdot)$ which minimizes cost functional (8)

The exact solution are: $u^{*}(t)=\left\{\begin{array}{l}-1,0 \leq t \leq \frac{\pi}{2} \\ 1, \frac{\pi}{2} \leq t \leq \frac{3 \pi}{2} \\ -1, \frac{3 \pi}{2} \leq t \leq 2 \pi\end{array}\right.$

Fig. 1 - Fig. 2 shows the comparison between numerical solution and approximate solution for $u_{0}=0.1$. Table 1 presents simulation results for different initial guess and accuracy of this problem.

Table 1: Simulation results for different initial guess and accuracy

\begin{tabular}{|c|c|c|c|c|c|c|}
\hline & $u_{0}$ & Accuracy & Error $\varepsilon_{u}$ & Error $\varepsilon_{x_{1}}$ & Error $\varepsilon_{x_{2}}$ & $I_{\min }$ \\
\hline 1 & 0 & 0,01 & - & - & - & - \\
\hline 2 & 0 & 0,001 & 0,998 & 1,666 & 1,703 & $-3,997$ \\
\hline 3 & 0,9 & 0,001 & 0,998 & 1,418 & 1,479 & $-3,997$ \\
\hline 4 & $-0,6$ & 0,001 & 0,998 & 1,646 & 1,413 & $-3,998$ \\
\hline 5 & $-0,9$ & 0,0001 & 0,0998 & 0,2428 & 0,2068 & $-3,9996$ \\
\hline 6 & 0,1 & 0,00001 & 0,00999 & 0,21223 & 0,20841 & $-3,99999$ \\
\hline
\end{tabular}




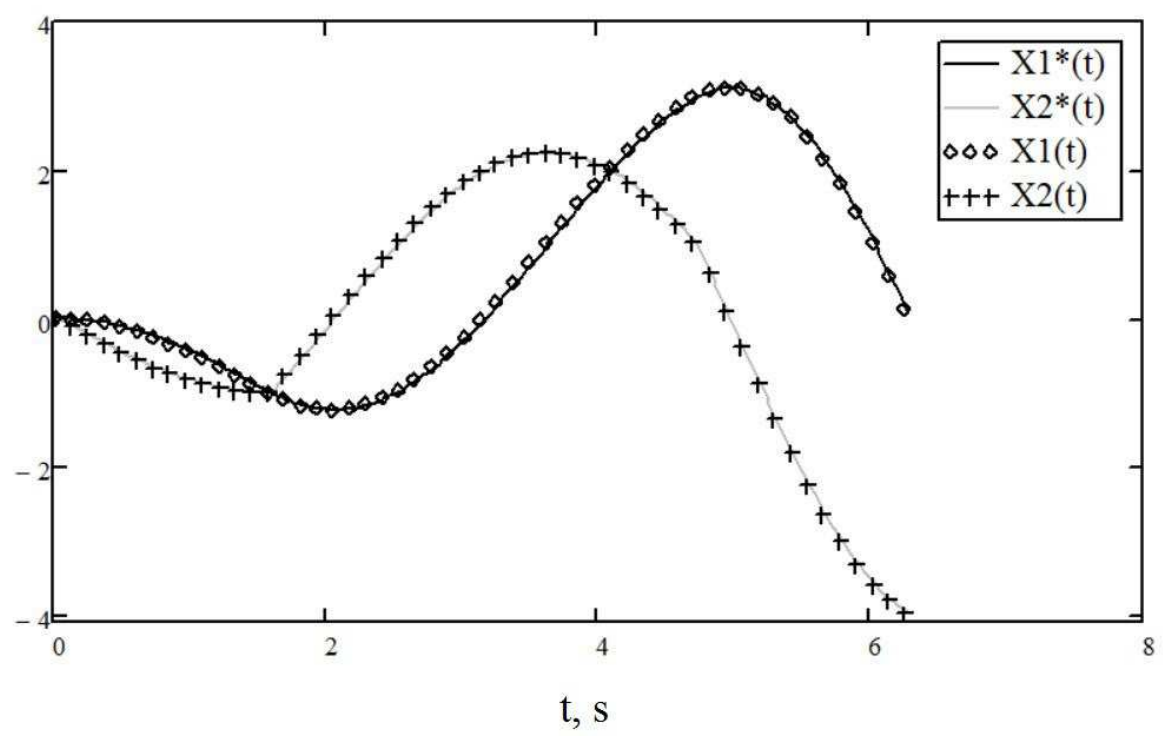

Figure 1: The suboptimal states

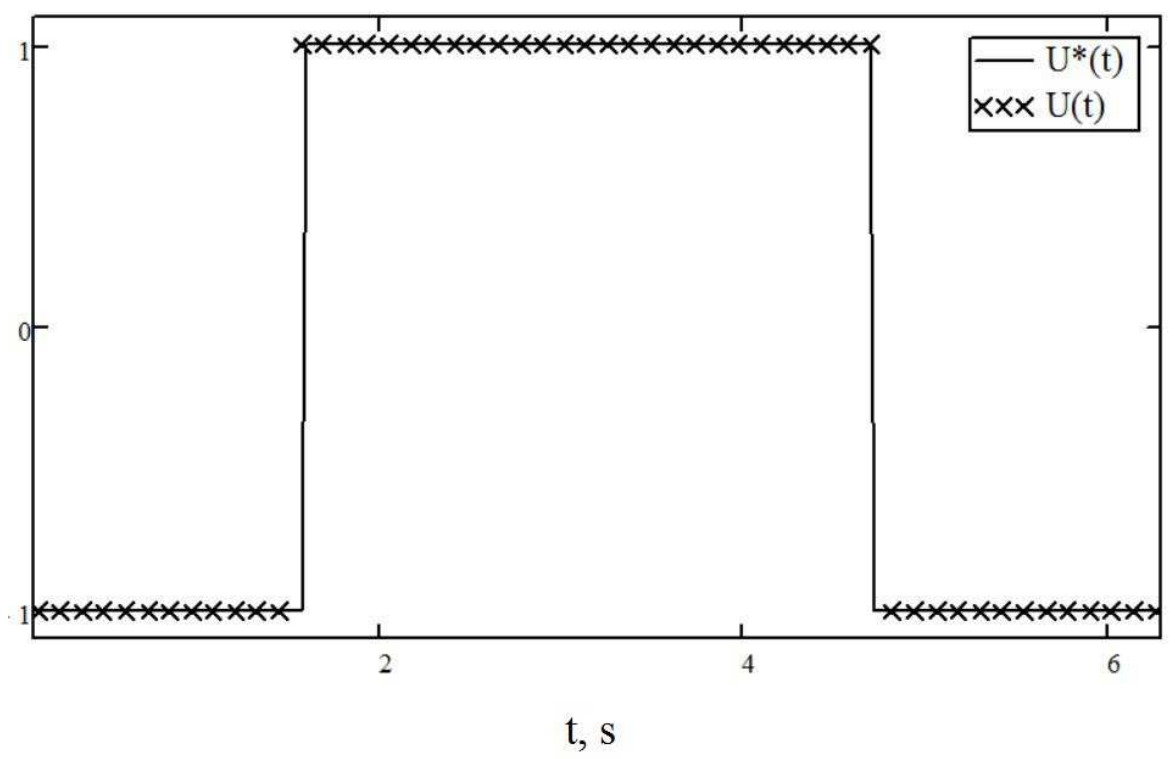

Figure 2: The suboptimal control 


\section{Conclusion}

Thus one of the first-order methods, which is called here the method of successive approximations and which is based on Pontryagin's maximum principle, is described in this paper. The existent experience shows that the method considered in this paper is rather simple, efficient, and reliable for a wide class of optimal control problems.

\section{References}

[1] Igor Grigoryev, Svetlana Mustafina, Global optimization of functions of several variables using parallel technologies, International Journal of Pure and Applied Mathematics, 106, No. 1 (2016), 301-306, doi: 10.12732/ijpam.v106i1.24.

[2] Gulnaz Shangareeva, Igor Grigoryev, Svetlana Mustafina, Comparative analysis of numerical solution of optimal control problems, International Journal of Pure and Applied Mathematics, 110, No. 4 (2016), 645-649, doi: 10.12732/ijpam.v110i4.6.

[3] Igor Grigoryev, Eldar Miftakhov, Svetlana Mustafina, Mathematical modelling of the copolymerization of styrene with maleic anhydride in a homogeneous environment, International Journal of Chemical Sciences, 14, No. 1 (2016), 381-386. 